\title{
Factors associated with access to health services and quality of life in knee osteoarthritis patients: a multilevel cross- sectional study
}

\author{
Siriwan Choojaturo ${ }^{1,2}$ (D) Siriorn Sindhu ${ }^{3^{*}}$, Ketsarin Utriyaprasit ${ }^{1}$ and Chukiat Viwatwongkasem ${ }^{2}$
}

\begin{abstract}
Background: The main purpose of health service systems is to improve patients' quality of life (QoL) and to ensure equitable access to health services. However, in reality, nearly half of knee osteoarthritis (OA) patients present to the health system do not have access to health services, and their QoL remains poor. These circumstances raise important questions about what (if any) factors can improve health care accessibility and QoL for knee OA patients.

Methods: A multicenter, cross-sectional survey was performed with 618 knee OA patients who received care at 16 hospitals in Thailand. Structural equation modeling (SEM) was conducted to investigate the association of health service factors and patient factors with access to health services and QoL.

Results: The QoL of knee OA patients was very poor (mean score $=33.8$ ). Only $2.1 \%$ of the knee OA patients found it easy to obtain medical care when needed. Approximately $39.4 \%$ of them were able to access appropriate interventions before being referred for knee replacement. More than 85\% of orthopedic health services had implemented chronic disease management (CDM) policy into practice. However, the implementation was basic, with an average score of 5.9. SEM showed that $\mathrm{QoL}$ was determined by both health system factors $(\beta=.10, p=.01)$ and patient factors $(\beta=.29$, $p=.00$ for self-management and $\beta=-.49, p=.00$ for disease factors). Access to health services was determined by selfmanagement $(\beta=.10, p=.01)$, but it was not significantly associated with $\mathrm{QoL}(\beta=.00, p=1.0)$.

Conclusions: This study provides compelling information about self-management, access to health services and QoL from the individual and health service system perspectives. Furthermore, it identifies a need to develop health services that are better attuned to the patient's background, such as socioeconomic status, disease severity, and self-management skills.
\end{abstract}

Keywords: Health service system, Osteoarthritis, Chronic disease management, Quality of life, Access, Self-management

\section{Background}

In large epidemiology studies, osteoarthritis (OA) has been reported as a major common cause of extremely poor quality of life (QoL) for hundreds of millions of adults of all ages worldwide [1]. The QoL of knee OA patients was found to be almost $50 \%$ lower than that of non-OA patients [2], and patients with end-stage OA

\footnotetext{
* Correspondence: siriorn.sin@mahidol.ac.th

${ }^{3}$ Mahidol University, Faculty of Nursing, 2 Wang Lang Road, Siriraj, Bangkoknoi, Bangkok 10700, Thailand

Full list of author information is available at the end of the article
}

reported a health state equal to or worse than that of patients with other chronic diseases [3]. OA has all the hallmarks of a harmful condition. It has been found to have the fourth greatest impact on the overall health of the world population in terms of both death and disability [4], highlighting the need for health system reforms.

In 2000, a worldwide campaign called the "Bone and Joint Decade" was launched to address this burden $[5,6]$. Additionally, the Institute of Medicine (IOM) has advocated the Chronic Disease Management (CDM) policy to redesign the health service system at all levels $[7,8]$. Since then, many countries, including Thailand, have established 
and implemented health policy, strategy, and planning at a national or institutional level. Subsequently, OA guidelines have been developed and implemented to provide a standard of care and assist physicians worldwide in tailoring OA management [9]. However, in reality, nearly half of knee OA patients in the health system do not have access to optimal health services, and their QoL remains immensely poor $[10,11]$, even in developed countries where health resources are plentiful.

Traditionally, physicians have had the authority to tell patients what to do and have expected patients to follow their orders. They have also tended to use management options such as medication and surgery for quick solutions, with a great focus on pain management [12, 13]. However, the actual provision of physician services remains limited. Safety, long waiting times, and health financing have been identified as problems [14]. Consequently, knee OA patients have tried to manage their care themselves, and they needed appropriate information and practical skills $[15,16]$. They desire to live well with their condition [17]. Therefore, responsibility for the management of OA overlaps among patients, providers, and health systems.

The prevalence of knee OA in developing countries, including Thailand, is generally similar to that seen in high-income countries (HIC) [18]. In addition, the feasibility of implementing a full multidisciplinary care team in Thailand given the restrictions of a limited health workforce and funding is low. A rational prediction is that the impact of the disease may be extreme because of the low general standard of living. However, the situation is more complex than this. The body of evidence substantiates that access to health services and QoL are complex constructs that are correlated with health service system factors and patient factors. However, a dearth of research on the topic is available. Furthermore, the effect of interplay of patient factors and health service system factors on access to health services and QoL has rarely been explored. To address this evidence gap, research is needed to determine whether certain factors can improve access to health services and QoL in knee OA patients.

\section{Methods}

\section{Study design and setting}

A multilevel, hospital-based survey was conducted at 16 hospitals in Thailand from June 2015 to June 2016 to determine how health service system factors and patient factors are associated with access to health services and QoL in patients who have been diagnosed with knee OA. A structural equation modeling (SEM) analysis was used to test the effect of the exogenous (health service system factors and patient factors) construct variables on the endogenous (access to health services and QoL) construct variables.

\section{Study participants}

The sample in this study consisted of 2 groups. One comprised 16 groups of health care providers from 16 hospitals. The other comprised 618 patients diagnosed with knee OA based on radiographic evidence and/or American College of Rheumatology clinical criteria.

\section{Sampling}

The researcher employed a proportionate stratified random sampling technique by service provider characteristics to obtain research participants.

In the first stage, 8 hospitals offering tertiary health services were selected by random sampling from a list of 56 hospitals in 34 provinces that followed the service plan of the Ministry of Public Health, Thailand [19]. The hospitals were eligible to participate if they were regional or universal hospitals, had orthopedists with specialty or subspecialty training in the areas of hip-knee treatment, and provided comprehensive, multidisciplinary care.

In the second stage, 8 hospitals offering primary health services were selected by random sampling from the list of hospitals within the same province as 8 hospitals offering tertiary health services. All the hospitals were eligible to participate if they were district or community hospitals and had general doctors and health care teams to provide generalist medical care and referred patients in need of more advanced conditions to tertiary health services. Consequently, 16 groups of healthcare providers who had relevant knowledge or expertise in orthopedic care and who were full-time regular employees were purposively selected from each hospital.

Finally, a total of 618 individual knee OA patients were selected who met the following eligibility criteria: (1) age 18 years and older; (2) a diagnosis of knee OA made 12 months or more before data collection began; and (3) kept at least $80 \%$ of appointments within the 12 months before their participation in the study. The patients were selected by random sampling from the total number of knee OA patients within each hospital. Individuals were excluded if they had cognitive dysfunction or demonstrated an unstable condition during the study (i.e., respiratory failure or unstable angina).

\section{Outcome measures Quality of life}

The Osteoarthritis Knee and Hip Health-related Quality of Life (OAKHQOL) is a disease-specific QoL questionnaire for patients with knee and hip OA [20]. It is selfadministered and consists of 43 items in five dimensions. The dimension scores are standardized from 0 (worst QoL) to 100 (best QoL). This QoL instrument has been used in several clinical and epidemiological studies [21]. The OAKHQOL has been shown to possess internal consistency, with alpha coefficients ranging from 0.80 
0.96, and a good test-retest result, with interclass correlation coefficients ranging from $0.73-0.87$. The construct and criterion validity have also been examined [22].

\section{Chronic disease management}

The Assessment of Chronic Illness Care (ACIC) is an international questionnaire used to evaluate how well a practice team or organization implements chronic disease management $(\mathrm{CDM})$ policy in practice [23]. The ACIC questionnaire consists of 32 items grouped into seven components. The component scores are standardized from 0 (no implementation) to 11 (full implementation). The ACIC has been shown to possess internal consistency, with alpha coefficients ranging from 0.85 0.97 , and good test-retest results, with interclass correlation coefficients ranging from $0.87-1.00$. The construct and criterion validity have also been examined [24].

\section{Access to health services}

The Osteoarthritis Quality Indicators (OAQIs) is a specific quality indicator used to measure whether standards of care are provided for patients with knee and hip OA based on medical records [25]. The OAQIs consist of 15 items that are broadly applicable to current international guidelines for the assessment of nonpharmacological and pharmacological management of OA. Each item is answered with one of two checklist options (do, do not do). The number of indicators passed (items answered "do") is divided by the number of eligible medical records and then multiplied by 100 . The results are reported as a QI pass rate ranging from 0 to 100 , with higher pass rate scores indicating greater access.

\section{Self-management (SM)}

The Osteoarthritis Self-Management Screening (OASMS) is a self-administered questionnaire used to measure selfmanagement skills; it was developed by researchers based on a literature review and the Perceived Medical Condition Self-Management Scales for patients with chronic disease [26]. The OASMS consists of 26 items in 4 dimensions: (1) perceived need; (2) self-efficacy; (3) knowledge of the skill; (4) and adherence to recommendations. Dimension scores are standardized from 0 (worst SM) to 100 (best SM). The internal consistency was tested, and alpha coefficients ranged from $0.82-0.93$; a good testretest result was determined, with interclass correlation coefficients ranging from $0.68-0.92$. The construct and criterion validity have also been examined.

\section{Data collection}

To collect data on patient factors and QoL, the 618 eligible patients were invited to complete a sociodemographic questionnaire, a clinical profile questionnaire, and the OAKHQOL questionnaire. To gather data on available hospital-based orthopedic services and the practicality of CDM implementation, the 16 groups of health care providers were invited to answer a hospital-based profile questionnaire and the ACIC.

Data regarding access to health services were extracted from the electronic and paper records of 618 patients, and access rates were measured in terms of 15 OAQIs.

\section{Ethics}

The study received ethical approval through the Mahidol University Institutional Review Board (Ref. no. IRB-NS2015/22.0303), which determined that the study posed no risks to participants. Informed consent was obtained from all individual participants included in the study.

Data collection was conducted in compliance with the Good Clinical Practices protocol and the Declaration of Helsinki. The participants received written and oral information about the study, and they provided their written informed consent prior to the baseline data collection.

\section{Results}

In total, 618 knee OA patients were included in the analyses. The response rate was $100 \%$. The mean age was 64.7 years (SD 8.6). Only 32.3\% had completed secondary school. Most (77.9\%) were employed, and $77.7 \%$ had a low income. More than two-thirds (68.3\%) of the participants had been diagnosed in the severe stage. The mean QoL was 33.8 (SD 12.7), while the mean SM was 35.1 (SD 5.3) (Table 1).

The access rate percentage was 54.2 (SD 14.1). Nonsteroidal anti-inflammatory drugs (NSAIDs) and/or other types of analgesics were prescribed to all knee OA patients. Only $39.4 \%$ of knee OA patients could access appropriate interventions before being referred for total knee replacement (Table 2).

There was a non-statistically significant difference in the mean of CDM policy implementation (all components) between the different levels of health services, with a total CDM policy implementation mean score of 5.9 (Table 3).

SEM showed that both health system factors $(\beta=.10)$ and patient factors $(\beta=.29$ for SM and $\beta=-.49$ for disease factors) were directly or indirectly associated with QoL. However, the association between access to health services and QoL was not statistically significant. SM played an important mediating role and was correlated with access to health services $(\beta=.13)$. This finding was robust across symptom severity and remained significant after other factors associated with QoL were adjusted for (Fig. 1).

\section{Discussion}

This study demonstrated that the majority of knee OA patients were of early retirement age and had 
Table 1 Sociodemographic and clinical characteristics of the study participants $(N=618)$

\begin{tabular}{llll}
\hline Sociodemographic characteristics & $N$ & $\%$ \\
\hline Age & Mean=64.7 (SD. = 8.6) & & \\
Education level & Primary & 418 & 67.6 \\
& Secondary & 90 & 14.6 \\
& Tertiary & 110 & 17.8 \\
& Unemployed & 138 & 22.3 \\
Occupation status & Employed & 480 & 77.7 \\
& None: lower than & 490 & 79.3 \\
Income status & minimum wage & & \\
& Minimum wage to average & 87 & 14.1 \\
& income & & \\
& High income & 41 & 6.6 \\
Stage of disease, as diagnosed & Mild & 63 & 10.2 \\
by doctor & Moderate & 133 & 21.5 \\
& Severe & 422 & 68.3 \\
Quality of life & Mean = 33.8 SD (12.7) & & \\
Self-management & Mean = 35.1 SD (5.3) & & \\
\hline
\end{tabular}

low socioeconomic status (SES). This finding could be attributed to economic factors as most elderly patients cannot afford to retire. It has been estimated that $80-90 \%$ of the population in low- and middleincome countries (LMIC) is involved in "heavy work". There is evidence that knee OA patients with

Table 2 Access to health services for the study participants $(N=618)$

\begin{tabular}{lll}
\hline Indicator & $\begin{array}{l}\text { Numerator/ } \\
\text { denominator }\end{array}$ & $\begin{array}{l}\text { Access rate } \\
(\%)\end{array}$ \\
\hline Access rate; mean (SD) & 54.2 (SD14.1) & \\
Nonpharmacological access rate & $353 / 618$ & 57.0 \\
$\quad$ Holistic assessment & $262 / 328$ & 79.9 \\
$\quad$ Weight control & $260 / 618$ & 42.1 \\
$\quad$ Exercise advice & $252 / 618$ & 40.8 \\
Health education & $45 / 284$ & 15.9 \\
$\quad$ Assistive devices & & \\
Pharmacological access rate & $331 / 618$ & 53.6 \\
$\quad$ Adequate acetaminophen use & $223 / 618$ & 37.7 \\
$\quad$ NSAIDs and COX-2 as adjuvant & & \\
analgesic drugs & $618 / 618$ & 100 \\
$\quad$ NSAIDs and/or other types of & & \\
analgesics & $215 / 335$ & 64.2 \\
NSAIDs plus PPI & $182 / 335$ & 54.9 \\
NSAID use with appropriate risk & & \\
assessment & $112 / 284$ & 39.4 \\
All applicable indicators before referral \\
for TKR
\end{tabular}

NSAIDs = nonsteroidal anti-inflammatory drugs; $C O X-2=$ cyclooxygenase 2; $P P I=$ proton-pump inhibitor; $T K R=$ total joint replacement a lower SES have a poorer QoL [27]. However, this study found that SES factors affected QoL not directly but through SM. This finding is similar to findings in many recent studies that revealed that SES alone is not associated with QoL in knee OA patients [28-30].

The basic finding of this study was that worsening knee OA symptoms were related to the deterioration of QoL $(\beta=-.49, p=.00)$. The QoL of knee OA patients appeared to be low. However, after symptom severity was adjusted for, the mean QoL scores in this study were closely linked to those found in the rest of the world [31-33]. This indicates that the QoL of knee OA patients is dependent on their disease severity rather than their country-specific context.

Additionally, this study found that knee OA patients with a higher SES might not have better access to health services. These data are inconsistent with those of previously published reports $[34,35]$. One reason for this discrepancy is that this study was conducted in a country where national health care use tends to favor the poor, especially since the implementation of a universal coverage policy in 2011 and a free medical care program for destitute elderly people in 2009. These two programs help facilitate equal access to health services for all Thais by removing cost barriers [36].

Most importantly, this study found that knee OA patients who had better SM not only had better QoL $(\beta=.29, p=.00)$ but also had better access to health services $(\beta=.18, \mathrm{p}=.00)$. This finding was robust across symptom severity levels and remained significant after many factors associated with QoL were adjusted for. Findings from qualitative studies have indicated that the majority of knee OA patients view individual responsibility as a key to maintaining health and living well with knee OA [13]. Conceivably, OA patients' desire to manage the impact of their condition on their life facilitates SM. Patients in a study by Miller et al. [37] reported that they attempted to adapt their lifestyle to reduce knee joint symptoms. Some patients took the initiative to start medication and nonpharmacological treatment on their own. Most of these patients sought information to gain control over their condition using a variety of sources, such as multimedia platforms and peers [38]. Therefore, they also had to access appropriate information to increase their knowledge how to self-manage their problem to improve QoL.

Interestingly, $\mathrm{SM}$ was strongly linked to patients' socioeconomic (SES) factors $(\beta=.28, p=.00)$ and disease factors $(\beta=.-43, \mathrm{p}=.00)$. Thus, QoL improved or worsened the effects of SES and disease factors on patients' ability to manage their own disease. The mechanisms for the association between SES and SM remain unclear. Indirect evidence suggests that SES plays a pivotal role in 
Table 3 CDM policy implementation categorized by level of health service $(N=16)$

\begin{tabular}{|c|c|c|c|c|c|}
\hline \multirow[t]{3}{*}{ CDM policy implementation } & \multicolumn{4}{|c|}{ Level of health service } & \multirow[t]{3}{*}{$p$-value } \\
\hline & \multicolumn{2}{|c|}{$\begin{array}{l}\text { Primary } \\
N=8\end{array}$} & \multicolumn{2}{|c|}{$\begin{array}{l}\text { Tertiary } \\
N=8\end{array}$} & \\
\hline & Mean & SD & Mean & SD & \\
\hline Health care organization & 6.5 & 3.1 & 7.4 & 1.6 & .51 \\
\hline Community resource & 5.2 & 2.7 & 5.5 & 3.7 & .88 \\
\hline Self-management support & 5.5 & 3.0 & 5.5 & 1.6 & 1.00 \\
\hline Decision support system & 5.4 & 3.0 & 5.4 & 2.0 & 1.00 \\
\hline Delivery system design & 6.0 & 3.1 & 6.0 & 1.3 & .97 \\
\hline Clinical information system & 6.0 & 3.3 & 6.1 & 1.2 & .93 \\
\hline Total CDM policy implementation; mean (SD) & \multicolumn{4}{|c|}{5.9 (SD 2.0) } & \\
\hline
\end{tabular}

$p=.05, \mathrm{CDM}=$ chronic disease management

the lives of citizens [39]. It is essential that patients with low SES receive sufficient knowledge to empower them to actively manage their health.

Unsurprisingly, knee OA patients attempt SM when their symptom severity increases. This finding contrasts with the findings of previous research showing that COPD patients who reported a higher symptom burden also reported lower SM [40]. There is a misbelief that knee OA is just part of the aging process. Knee OA patients might simply be told that there is nothing to be done except knee arthroplasty [41]. Although health care professionals state that SM is useful in improving patients' health outcomes, it is often completely under recognized and omitted from patient education [42, 43]. Thus, knee OA patients are left underprepared for SM when their symptom severity is mild. Furthermore, most health care providers have focused on SM-related support rather than on patient SM. However, a recent review reported that SM support alone likely has little to no effect on improving knee OA patients' health outcomes [44].

Knee OA patients have expressed disappointment that the public health care system does little to help them [45]. Given this reality, to improve patients' SM, a better understanding of how to deliver health services and align evidence with policy and practice is required. We must also reshape our mindsets and imagine ourselves as patients, particularly those with SES disadvantages. Increasing awareness among the public and clinicians regarding the multifaceted impact of $\mathrm{OA}$ on the lives of a large segment of the population may increase attention to the problem and elicit more proactive management by both patients and physicians.

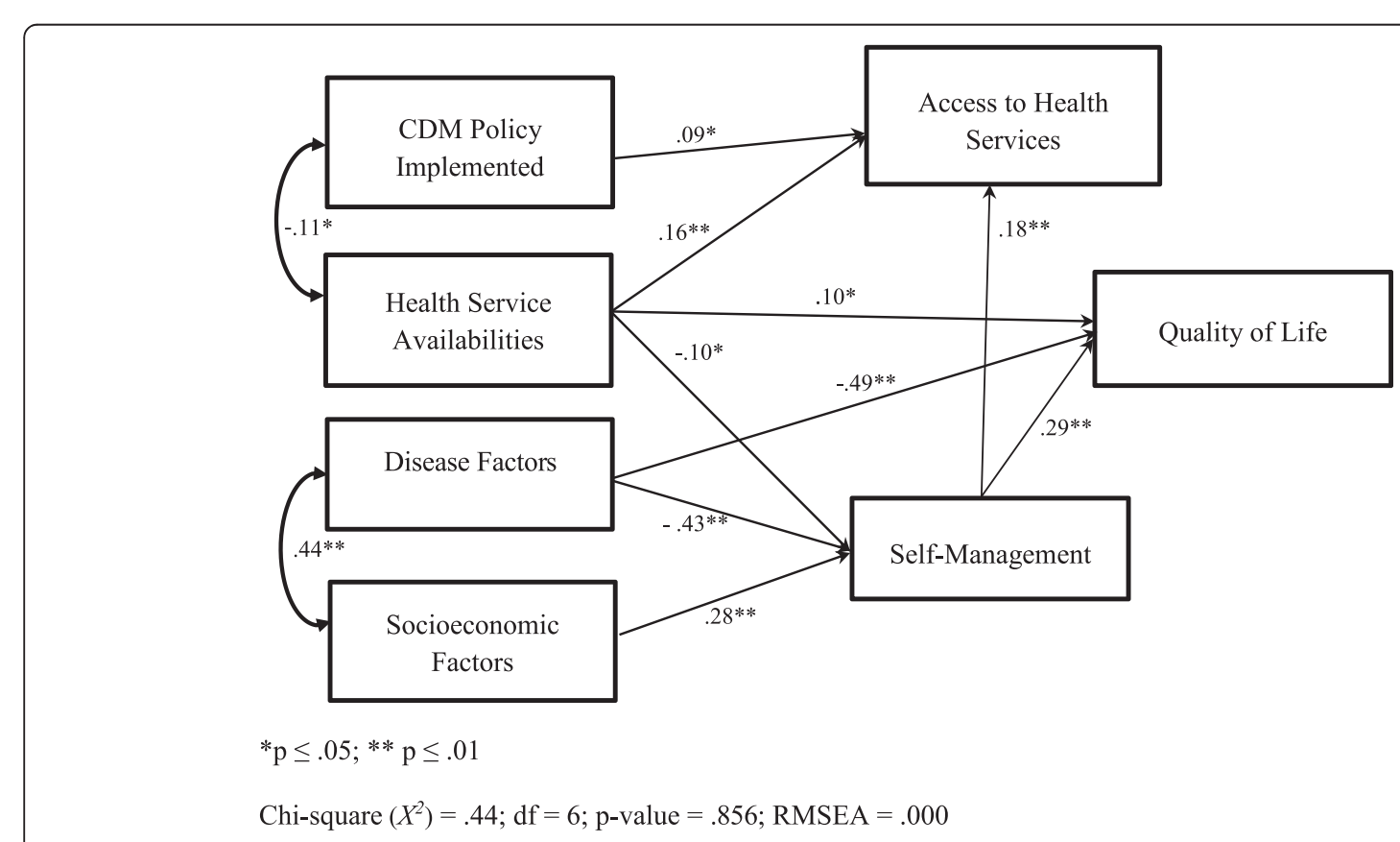

Fig. 1 A modified model of the study 
The present study has several limitations. First, it was conducted in government hospital settings with specific recruitment requirements. Thus, it cannot be extrapolated to other settings (private hospitals, home health care, populations with other ethnic backgrounds, etc.). The data were collected in a single country. Therefore, the results of this study may not always be extrapolated to countries with different health care policies. This was not a placebocontrolled trial; thus, the extent to which QoL improvements may have been related to placebo effects cannot be estimated. Other limitations of the study are that the access rates may not reflect the care quality, as perceived by the patients, and health care providers may interpret the quality differently from their patients. However, self-reports might represent a weakness because there is potential for recall bias, which might have led to over- or underestimated access rates in the study.

A key strength of this study of knee OA patients is that it provides powerful information about access to health services and QoL from the individual and health system perspectives. One of the first steps toward improving QoL in adults with OA is to develop a better understanding of the factors that coexist with and influence QoL. Most studies focus on single variables concerned with individual factors (e.g., gender, education level, income, career and severity of disease), while QoL involves multiple factors. This study strongly supports the notion that SM is independently correlated with improved QoL. Therefore, understanding patient SM is important to identifying other factors for best practice and potential strategies to create more patient-centered health services for OA care.

\section{Conclusions}

This study highlights the strengths of existing OA clinical practices and the opportunity to create health service systems that positively influence future care. Health service system factors and patient factors all appear to influence QoL in knee OA patients. Most importantly, the effective management of OA care begins and ends with the patients. Moreover, there is a need to target research efforts toward specific interventions to improve patient SM, which remains a key patient factor.

\section{Abbreviations}

CDM: Chronic disease management; OA: Osteoarthritis; QoL: Quality of life; SES: Socioeconomic status; SM: Self-management

\section{Acknowledgements}

We are extremely grateful to all study participants. The authors also extend their special thanks to all hospital policy directors, data facilitators and collaborators.

\section{Authors' contributions}

All authors made substantial contributions to the conception, study design and acquisition of data. SC, SS and CV analyzed and interpreted the data. SC, $\mathrm{SS}$ and $\mathrm{KU}$ were involved in drafting and revising the manuscript. All authors read and approved the final manuscript. Each author has agreed to be accountable for all aspects of the work.

\section{Authors' information \\ Siriwan Choojaturo, D.N.S. (candidate), RN \\ Siriorn Sindhu, PhD, has expertise in women's health and health system research. \\ Ketsarin Utriyaprasit, PhD, has expertise in adult education, geriatrics, and medical science nursing. \\ Chukiat Viwatwongkasem, PhD, has expertise in probability theory, \\ biostatistics, and ad.}

\section{Funding}

The research was self-funded. The researchers received their normal government salary from Mahidol University and Police General Hospital by virtue of their positions. The institute and organization played no role in the design, execution, analysis or interpretation of the data or in the writing up of the findings.

\section{Availability of data and materials}

Data are available upon reasonable request from the corresponding author. The raw data that support the finding of this study are available in the [figshare] repository with the identifier [DOI: https://doi.org/10.6084/m9. figshare.7784792].

Ethics approval and consent to participate

Ethical approval for the study was obtained from the Mahidol University Institutional Review Board (MU-IRB) (Ref. no. IRB-NS-2015/22.0303). Written informed consent was obtained from all study participants.

\section{Consent for publication}

Not applicable.

\section{Competing interests}

The authors declare that they have no competing interests.

\section{Author details}

'Department of Surgical Nursing, Faculty of Nursing, Mahidol University, Bangkok 10700, Thailand. ${ }^{2}$ Department of Biostatistics, Faculty of Public Health, Mahidol University, Bangkok 10400, Thailand. ${ }^{3}$ Mahidol University, Faculty of Nursing, 2 Wang Lang Road, Siriraj, Bangkoknoi, Bangkok 10700, Thailand.

Received: 17 May 2018 Accepted: 20 August 2019

Published online: 11 October 2019

\section{References}

1. Cross M, Smith E, Hoy D, Nolte S, Ackerman I, Fransen M, et al. The global burden of hip and knee osteoarthritis: estimates from the global burden of disease 2010 study. Ann Rheum Dis. 2014;73:1323-30.

2. Ackerman $\mathrm{IN}$, Bennell $\mathrm{KL}$, Osborne $\mathrm{RH}$. Decline in health-related quality of life reported by more than half of those waiting for joint replacement surgery: a prospective cohort study. BMC Musculoskelet Disord. 2011;12:108.

3. Nyvang J, Hedstrom M, Gleissman SA. It's not just a knee, but a whole life: a qualitative descriptive study on patients' experiences of living with knee osteoarthritis and their expectations for knee arthroplasty. Int J Qual Stud Health Well-being. 2016;11:30193.

4. Park Jl, Jung HH. Estimation of years lived with disability due to noncommunicable diseases and injuries using a population-representative survey. PLoS One. 2017;12:e0172001.

5. Woolf AD, Erwin J, March L. The need to address the burden of musculoskeletal conditions. Best Pract Res Clin Rheumatol. 2012;26:183-224.

6. Mody GM, Brooks PM. Improving musculoskeletal health: global issues. Best Pract Res Clin Rheumatol. 2012;26:237-49.

7. Brand CA, Ackerman IN, Tropea J. Chronic disease management: improving care for people with osteoarthritis. Best Pract Res Clin Rheumatol. 2014;28:119-42. 
8. Brand CA, Ackerman IN, Bohensky MA, Bennell KL. Chronic disease management: a review of current performance across quality of care domains and opportunities for improving osteoarthritis care. Rheum Dis Clin N Am. 2013;39:123-43.

9. Ferreira de Meneses S, Rannou F, Hunter DJ. Osteoarthritis guidelines: Barriers to implementation and solution. Ann Phys Rehabil Med. 2016; 59:170-3

10. Hagen KB, Smedslund G, Osteras N, Jamtvedt G. Quality of communitybased osteoarthritis care: a systematic review and meta-analysis. Arthritis Care Res. 2016;68:1443-52.

11. Postler A, Ramos AL, Goronzy J, Gunther KP, Lange T, Schmitt J, et al. Prevalence and treatment of hip and knee osteoarthritis in people aged 60 years or older in Germany: an analysis based on health insurance claims data. Clin Interv Aging. 2018;13:2339-49.

12. Gruber $\mathrm{WH}$, Hunter DJ. Transforming osteoarthritis care in an era of health care reform. 2010;26:433-44.

13. Erwin J, Edwards K, Woolf A, Whitcombe S, Kilty S. Better arthritis care: patients' expectations and priorities, the competencies that communitybased health professionals need to improve their care of people with arthritis? Musculoskeletal Care. 2018;16:60-6.

14. Charlesworth J, Fitzpatrick J, Perera NKP, Orchard J. Osteoarthritis- a systematic review of long-term safety implications for osteoarthritis of the knee. BMC Musculoskelet Disord. 2019;20:151.

15. Jinks C, Ong BN, O'Neill T. "Well, it's nobody's responsibility but my own." A qualitative study to explore views about the determinants of health and prevention of knee pain in older adults BMC Public Health 2010; 10:148.

16. Carmona-Teres V, Moix-Queralto J, Pujol-Ribera E, Lumillo-Gutierrez I, Mas X,

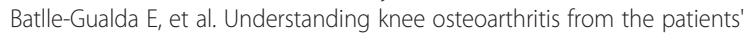
perspective: a qualitative study. BMC Musculoskelet Disord. 2017;18:225.

17. Smith TO, Purdy R, Lister S, Salter C, Fleetcroft R, Conaghan P. Living with osteoarthritis: a systematic review and meta-ethnography. Scand J Rheumatol 2014:43:441-452.

18. Tangtrakulwanich B, Suwanno P. Epidemiology and risk factors of patellofemoral osteoarthritis in adults: a population-based study in southern Thailand. J Med Assoc Thail. 2012;95:1048-52.

19. Tangcharoensathien V, Witthayapipopsakul W, Panichkriangkrai W Patcharanarumol W, Mills A. Health systems development in Thailand: a solid platform for successful implementation of universal health coverage. Lancet. 2018;391:1205-23.

20. Rat AC, Coste J, Pouchot J, Baumann M, Spitz E, Retel-Rude N, et al. A new instrument to measure quality of life in knee and hip osteoarthritis. J Clin Epidemiol. 2005;58:47-55

21. Guillemin F, Rat AC, Roux CH, Fautrel B, Mazieres B, Chevalier X, et al. The KHOALA cohort of knee and hip osteoarthritis in France. Joint Bone Spine. 2012;79:597-603.

22. Goetz C, Ecoss E, Rat AC, Pouchot J, Coste J, Guillemin F. Measurement properties of the osteoarthritis of knee and hip quality of life OAKHQOL questionnair: an item response theory analysis. Rheumatology(Oxford). 2011; 50:500-5.

23. Gomutbutra P, Aramrat A, Sattapansri W, Chutima S, Tooprakai D, Sakarinkul $P$, et al. Reliability and validity of a Thai version of assessment of chronic illness care (ACIC). J Med Assoc Thail. 2012;95:1105-13.

24. Cramm JM, Strating MM, Tsiachristas A, Nieboer AP. Development and validation of a short version of the assessment of chronic illness care (ACIC) in Dutch disease management programs. Health Qual Life Outcomes. 2011;9:49.

25. Edwards JJ, Khanna M, Jordan KP, Jordan JL, Bedson J, Dziedzic KS. Quality indicators for the primary care of osteoarthritis: a systematic review. Ann Rheum Dis. 2015;74:490-8.

26. Wallston KA, Rothman RL, Cherrington A. Psychometric properties of the Percieved diabetes self-management scales (PDSMS). J Behav Med. 2007:30:395-401.

27. Kiadaliri AA, De Verdier MG, Turkiewicz A, Lohmander LS, Englund M. Socioeconomic inequalities in knee pain, knee osteoarthritis, and healthrelated quality of life: a population-based cohort study in southern Sweden. Scand J Rheumatol. 2017:46:143-51.

28. Mosallanezhad Z, Sotoudeh GR, Jutengren G, Salavati M, Harms-Ringdahl K, Wikmar LN, et al. A structural equation model of the relation between socioeconomic status, physical activity level, independence and health status in older Iranian people. Arch Gerontol Geriatr. 2017:70:123-9.
29. Xie F, Kovic B, Jin X, He X, Wang M, Silvestre C. Economic and humanistic burden of osteoarthritis: a systematic review of large sample studies. Pharmacoeconomics. 2016:34:1087-100.

30. Espinosa R, Arce A, Esquivel JA, Alvarez E, Vega D, Torres JF, et al. Risk factors associated to the quality of life of patients with osteoarthritis in Mexico The impactar registry. Osteoarthr Cartil. 2017;25:S205.

31. Muraki S, Akune T, Oka H, En-Yo Y, Yoshida M, Saika A, et al. Association of radiographic and symptomatic knee osteoarthritis with health-related quality of life in a population-based cohort study in Japan: the ROAD study. Osteoarthr Cartil. 2010;18:1227-34.

32. Palo N, Chandel SS, Dash SK, Arora G, Kumar M, Biswal MR. Effects of osteoarthritis on quality of life in elderly population of Bhubaneswar, India: a prospective multicenter screening and therapeutic study of 2854 patients. Geriatr Orthop Surg Rehabil. 2015;6:269-75.

33. Wilson R, Blakely $T$, Abbott JH. Radiographic knee osteoarthritis impacts multiple dimensions of health-related quality of life: data from the osteoarthritis initiative. Rheumatology. 2018. https://doi.org/10.1093/rheumatology/key008.

34. Guillemin F, Carruthers E, Li LC. Determinants of MSK health and disability social determinants of inequities in MSK health. Best Pract Res Clin Rheumatol. 2014;28:411-33.

35. Cai J, Coyte PC, Zhao H. Decomposing the causes of socioeconomic-related health inequality among urban and rural populations in China: a new decomposition approach. Int J Equity Health. 2017;16:128.

36. Kanchanachitra C, Podhisita C, Archavanitkul K, Chamchan C, Siriratmongkol K, Jaratsit S, et al. Thai health 2013: Thailand reform: restructuring power, empowering citizens. Nakhon Pathom: Institute for Population and Social Research, Mahidol University; 2014

37. Miller JL, Teare SR, Marlett N, Shklarov S, Marshall DA. Support for living a meaningful life with osteoarthritis: a patient-to-patient research study. Patient. 2016;9:457-64

38. Wluka A, Chou L, Briggs A. Understanding the needs of consumers with musculoskeletal conditions: consumers' perceived needs of health information, health services and other non-medical services: a systematic scoping review. MOVE Muscle Bone \& Joint Health: Melbourne; 2016.

39. Papandony MC, Chou L, Seneviwickrama M, Cicuttini FM, Lasserre K Teichtahl AJ, et al. Patients' perceived health service needs for osteoarthritis (OA) care: a scoping systematic review. Osteoarthr Cartil. 2017;25:1010-25.

40. Ainee K, Andrew PD, Peymanee A, Racheal EJ. Self-management behavior and support among primary care COPD patients: cross-sectional analysis of data from Birmingham chronic obstructive pulmonary disease cohort. NPJ Pim Care Respir Med. 2017;27:1-10.

41. Paskins Z, Sanders T, Hassell AB. Comparison of patient experiences of the osteoarthritis consultation with GP attitudes and beliefs to OA: a narrative review. BMC Fam Pract. 2014;15:46

42. Ali F, Jinks C, Ong BN. "...Keep mobile, I think that's half the battle." A qualitative study of prevention of knee pain in symptomless older adults. BMC Public Health 2012;12:753.

43. Selten EM, Geenen R, van der Laan WH, van der Meulen-Dilling RG, Schers HJ, Nijhof MW, et al. Hierarchical structure and importance of patients' reasons for treatment choices in knee and hip osteoarthritis: a concept mapping study. Rheumatology. 2017;56:271-8.

44. van der Burg LR KFP, Buchbinder R, Osborne RH, Johnston RV, Pitt V. Selfmanagement education programmes for osteoarthritis. Cochrane Database Syst Rev. 2014;5:Cd008963.

45. Walsh L, Hill S, Wluka AE, Brooks P, Buchbinder R, Cahill A, et al. Harnessing and supporting consumer involvement in the development and implementation of models of care for musculoskeletal health. Best Pract Res Clin Rheumatol. 2016;30:420-44.

\section{Publisher's Note}

Springer Nature remains neutral with regard to jurisdictional claims in published maps and institutional affiliations. 